\title{
Rôle clé de la kinase eEF2K dans la survie cellulaire à la privation de nutriments
}

\section{Agir sur le facteur d'élongation de la traduction eEF2}

Gabriel Leprivier ${ }^{1}$, Barak Rotblat ${ }^{1}$, Olivier Delattre ${ }^{2}$, Poul H. Sorensen ${ }^{1}$

Rôles des kinases ęF2K et AMPK dans l'adaptation cellulaire à la privation de nutriments

La privation de nutriments représente un stress physiologique menaçant la survie des cellules. Un certain nombre de mécanismes moléculaires ont émergé durant l'évolution afin de préserver l'intégrité cellulaire lors d'un stress nutritif. C'est le cas de la protéine kinase activée par l'AMP (ou AMPK), qui a pour fonction de détecter et de réguler le niveau énergétique intracellulaire [11]. Cette kinase est activée lorsque les rapports intracellulaires AMP/ATP ou ADP/ATP s'élèvent, ce qui signale un stress énergétique [1]. II en résulte l'inhibition de processus anaboliques, tels que la prolifération et la traduction, et l'activation de processus cataboliques, tels que la dégradation des acides gras, qui permettent de préserver l'homéostasie énergétique cellulaire (Figure 1) [1]. Un autre régulateur fondamental de la balance énergétique intracellulaire est la protéine kinase mTOR (mammalian target of rapamycin), cible de la rapamycine, qui coordonne les niveaux de prolifération et de synthèse protéique en fonction de la disponibilité en acides aminés et en ATP (Figure 1 ) $[2,3,12]$.

Une limitation du niveau des nutriments est caractéristique de pathologies telles que le cancer. En effet, les cellules cancéreuses ont un accès restreint aux nutriments en raison d'ano- malies du réseau vasculaire tumoral [4]. De plus, et de manière paradoxale, de nombreuses oncoprotéines, telles que C-MYC, AKT (protéine kinase B) et mTOR, rendent les cellules hypersensibles à l'absence de nutriments [5-7]. Ceci illustre la nécessité absolue pour les cellules tumorales de développer des mécanismes d'adaptation pour survivre dans des conditions de carence nutritive. À ce jour, notre compréhension de ces mécanismes reste lacu-
${ }^{1}$ Département d'oncologie moléculaire, Centre de recherche contre le cancer de Colombie Britannique et département de pathologie, Université de Colombie Britannique, 675 West $10^{\text {th }}$ avenue, V5Z1L3 Vancouver, Canada ;

${ }^{2}$ Génétique et biologie des cancers, Inserm U830, Institut Curie, 26, rue d'UIm 75248 Paris Cedex 05, France. gabriel.leprivier@gmail.com

naire, alors qu'elle devrait permettre d'identifier de nouvelles cibles thérapeutiques.

Notre étude récente publiée dans Cell a permis de mettre à jour le rôle clé de la kinase eEF2K (eukaryotic translation elongation factor 2 kinase) dans la protection cellulaire contre la privation de nutriments [8]. Cette kinase est responsable de la phosphorylation et de l'inactivation du facteur d'élongation de la traduction eEF2, son unique substrat [9].

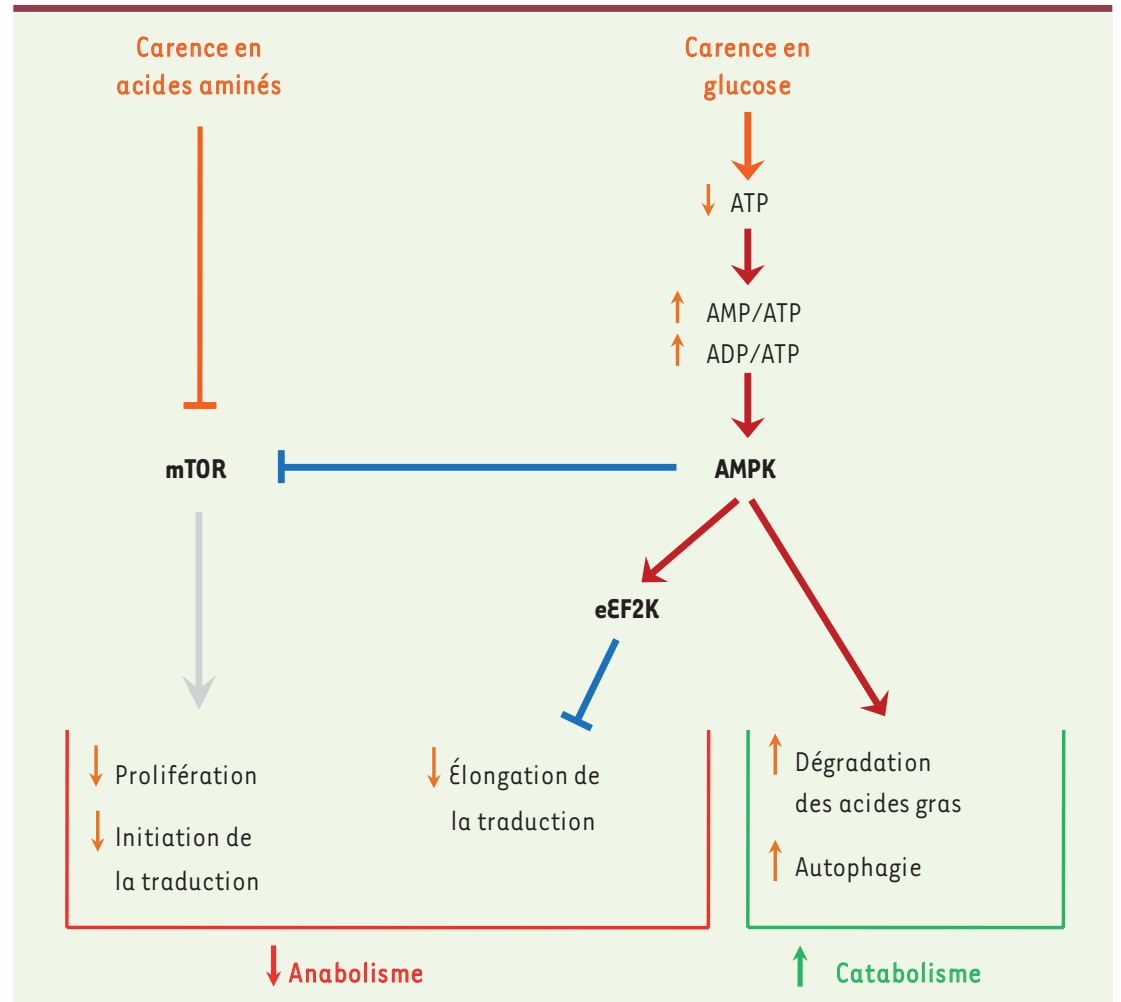

Figure 1. Principales voies de signalisation contrôlant la réponse à la privation de nutriments. 

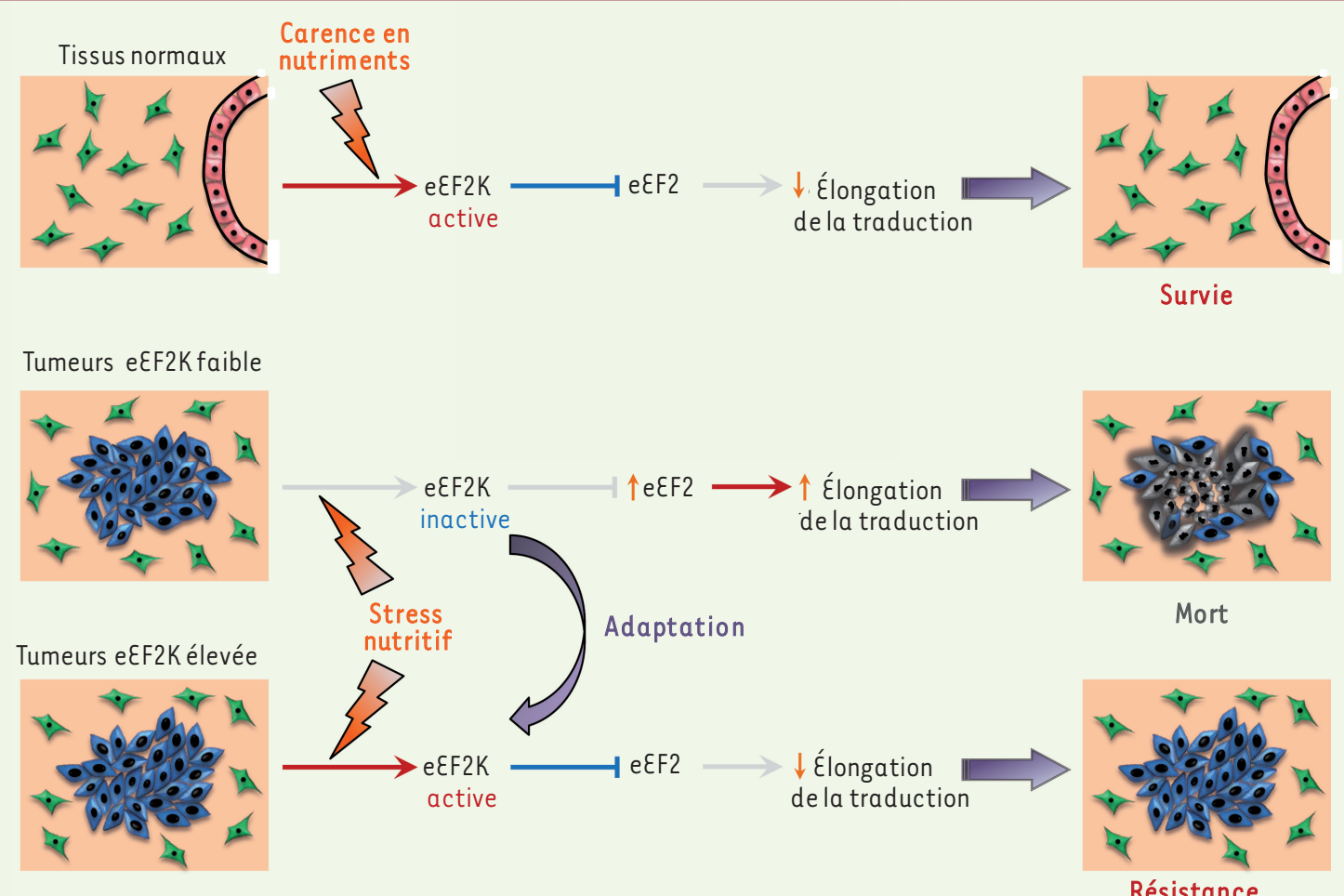

Résistance

Figure 2. Modèle pour le rôle de eEF2K dans la réponse biologique à la privation de nutriments dans les tissus normaux et cancéreux.

En réponse à un déficit en nutriments, eहF2K est activée par AMPK, aboutissant à l'inhibition de la traduction à l'étape d'élongation qui est sous le contrôle de eEF2 (Figure 1) [10]. Cette réponse biologique à la privation de nutriments est cruciale pour le maintien de l'homéostasie énergétique cellulaire, étant donné que la synthèse protéique est un des processus cellulaires les plus coûteux énergétiquement.

Dérégulation de eqF2 et de la traduction dans les cellules transformées soumises à un stress nutritif

Nos observations initiales ont été établies à l'aide d'un modèle de fibroblastes murins transformés par les oncoprotéines K-Ras ${ }^{\mathrm{V} 12}$ ou ETV6-NTRK3 (neurotrophic tyrosine kinase receptor, type 3). De façon remarquable, l'absence de nutriments induit l'apoptose des cellules transformées, mais n'affecte pas les cellules contrôles (non transformées). Parmi les paramètres étudiés (prolifé- ration, radicaux libres, autophagie et activité de mTOR), seules les activités des kinases AMPK et eEF2K étaient dérégulées dans les cellules transformées privées de nutriments, comparativement aux cellules normales. Ainsi, l'activité de ęF2K dans les cellules transformées reste faible en l'absence de nutriments, alors qu'elle est fortement induite dans les cellules normales. Ceci est dû au défaut d'activité d'AMPK dans les cellules transformées privées de nutriments, qui s'explique par un niveau élevé d'ATP; de ce fait, les rapports AMP/ATP et ADP/ ATP n'augmentent pas, indiquant paradoxalement une absence de stress énergétique. En conséquence, l'activité de eEF2 reste élevée en l'absence de nutriments dans les cellules transformées (par manque de l'activité inhibitrice de eEF2K), contribuant au maintien du taux d'élongation de la traduction, alors que, dans ces conditions, celui-ci est réduit dans les cellules normales. La dérégulation de eEF2 et de l'élongation de la traduction joue un rôle primordial dans I'hypersensibilité des cellules transformées à la privation de nutriments, puisque l'inhibition de eEF2 ou la surexpression de eEF2K (bloquant eદF2) dans ces cellules suffit à augmenter leur survie en absence de nutriments. II est possible que ceci soit dû à une réduction sélective de la synthèse de certaines protéines impliquées dans des processus anaboliques ou dans l'exécution de l'apoptose. Il est intéressant qu'une sous-population de cellules transformées résistantes à la privation de nutriments ait pu être isolée par un processus de sélection. Dans ces cellules, on observe une réactivation des kinases AMPK et ęF2K en réponse à I'absence de nutriments, comparativement aux cellules parentales, qui résulte de la ré-augmentation des rapports AMP/ ATP et ADP/ATP. L'inhibition d'AMPK ou de eहF2K compromet la survie des cellules sélectionnées en absence de nutriments, démontrant le rôle de eEF2K et du contrôle de l'élongation de la traduction 
dans l'adaptation des cellules transformées à la privation de nutriments.

\section{Impact de eEF2K dans l'adaptation} des cellules tumorales à la privation de nutriments in vivo

L'importance de eEF2K pour la survie des cellules tumorales a pu être démontrée in vivo dans des souris nude en déterminant l'impact d'une restriction calorique sur la croissance de tumeurs surexprimant ou non cette kinase. Ainsi, les tumeurs établies à partir des cellules transformées parentales (non sélectionnées) sont particulièrement sensibles à la restriction calorique, qui réduit leur croissance de moitié. II s'y associe une nécrose importante au sein de ces tumeurs. Or, la surexpression de eEF2K rend les tumeurs résistantes à la restriction calorique en bloquant l'induction de nécrose, permettant la poursuite de leur croissance. II apparaît donc que in vivo, eहF2K est primordiale pour la survie des cellules tumorales confrontées à un déficit en nutriments.

Ces résultats suggéraient que eहF2K pourrait être dérégulée dans les tumeurs humaines, permettant de promouvoir leur survie en conditions de stress nutritif. De fait, l'ARNm codant pour eEF2K est surexprimé dans des tumeurs agressives, comme les médulloblastomes et glioblastomes, comparativement aux tissus normaux. De plus, le niveau des transcrits eEF2K est un facteur de mauvais pronostic chez les patients atteints de ces deux types de cancer. Enfin, si l'activité kinase de eहF2K est particulièrement élevée au sein des tissus tumoraux dans les médulloblastomes, elle n'est pas détectée dans les tissus normaux environnants. L'ensemble de ces données montrent qu'en augmentant l'expression ou l'activité de eहF2K, les tumeurs acquièrent une protection contre le stress nutritif, suggérant un nouveau mécanisme d'adaptation des tumeurs (Figure 2).

Enfin, nous avons caractérisé la fonction biologique de eEF2 $K$ en conditions physiologiques de carence nutritive en utilisant l'organisme modèle $C$. elegans. Le gène codant pour eहF2 $K$ est en effet conservé chez $C$. elegans, dénommé efk-1, et son expression est induite par la privation de nutriments. En conditions nutritives normales, la durée de vie des vers sauvages et déficients pour efk-l est comparable. Néanmoins, en l'absence de nutriments, les vers déficients pour efk-l sont gravement affectés, leur durée de vie étant réduite de moitié par rapport aux organismes sauvages. Ces résultats démontrent que la fonction biologique de eहF2K est conservée au cours de l'évolution (Figure 2).

$\varepsilon n$ conclusion, notre étude a mis en évidence que eहF2K est un régulateur fondamental de la réponse biologique à la privation de nutriments, et que certaines tumeurs exploitent ce mécanisme afin de promouvoir leur adaptation au manque chronique de nutriments. $\diamond$ Surviving nutrient deprivation by restraining translation elongation: biological function of the eEF2 kinase

\section{REMERCIEMENTS}

Nous tenons à remercier l'ensemble de nos collaborateurs ayant contribué à cette étude.

\section{LIENS D'INTÉRÊT}

Les auteurs déclarent n'avoir aucun lien d'intérêt concernant les données publiées dans cet article.

\section{RÉFÉRENCES}

1. Hardie DG. AMP-activated protein kinase: an energy sensor that regulates all aspects of cell function. Genes Dev 2011 ; 25 : 1895-908.

2. Hay N, Sonenberg N. Upstream and downstream of mTOR. Genes Dev $2004 ; 18$ : 1926-45.

3. Zoncu R, Efeyan A, Sabatini DM. mTOR: from growth signal integration to cancer, diabetes and ageing. Nat Rev Mol Cell Biol 2011; 12 : 21-35.

4. Nagy JA, Chang SH, Dvorak AM, Dvorak HF. Why are tumour blood vessels abnormal and why is it important to know? BrJ Cancer 2009; 100 : 865-9.

5. Buzzai M, Bauer DE, Jones RG, et al. The glucose dependence of Akt-transformed cells can be reversed by pharmacologic activation of fatty acid betaoxidation. Oncogene $2005 ; 24: 4165-73$.

6. Choo AY, Kim SG, Vander Heiden MG, et al. Glucose addiction of TSC null cells is caused by failed mTORCl-dependent balancing of metabolic demand with supply. Mol Cell 2010 ; $38: 487-99$.

7. Shim H, Chun YS, Lewis BC, Dang CV. A unique glucosedependent apoptotic pathway induced by c-Myc. Proc Natl Acad Sci USA 1998 ; 95 : 1511-6.

8. Leprivier G, Remke M, Rotblat B, et al. The eहF2 kinase confers resistance to nutrient deprivation by blocking translation elongation. Cell 2013 ; 153 : 1064-79.

9. Ryazanov AG, Shestakova EA, Natapov PG. Phosphorylation of elongation factor 2 by $\varepsilon F-2$ kinase affects rate of translation. Nature $1988 ; 334: 170-3$.

10. Browne GJ, Finn SG, Proud CG. Stimulation of the AMP-activated protein kinase leads to activation of eukaryotic elongation factor 2 kinase and to its phosphorylation at a novel site, serine 398. J Biol Chem $2004 ; 279$ : 12220-31.

11. Foretz M, Taleux N, Guigas B, et al. Régulation du métabolisme énergétique par l'AMPK. Med Sci (Paris) $2006 ; 22: 381-8$.

12. Julien LA, Roux P. mTOR, la cible fonctionnelle de la rapamycine. Med Sci (Paris) 2010 ; 26 : 1056-60.

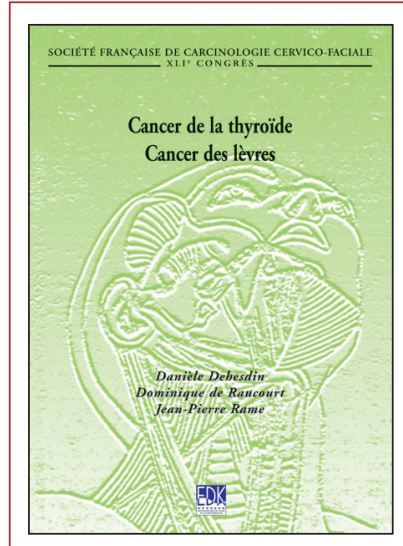

ISBN : 978-2-8425-4137-8 264 pages
Bon de commande à retourner à EDK, 25, rue Daviel - 75013 Paris

Tél. : 0158101905 - Fax : 0143293262 - E-mail : edk@edk.fr

NOM :

Prénom :

Adresse :

Code postal :

Ville :

Pays :

Fonction :

Je souhaite recevoir l'ouvrage Cancer de la thyroïde - Cancers des lèvres : $35 €+3 €$ de port $=38 €$ TTC

en ................. exemplaire, soit un total de ..................................... €

$\square$ Par chèque, à l'ordre de $\mathbf{E} \mathbf{D} \mathbf{K}$

$\square$ Par carte bancaire : $\square$ Visa $\square$ Eurocard/Mastercard

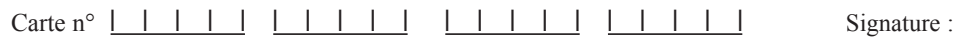

Date d'expiration: $\quad 1 \quad 1 \quad 1 \quad 1 \quad 1$

$\mathrm{N}^{\circ}$ de contrôle au dos de la carte : $\quad$ । $\quad$ । $\quad$ | 